\title{
Competencias sociales y tecnologías de la información y la comunicación como factores asociados al desempeño en estudiantes de básica primaria con experiencia de desplazamiento forzado*
}

\section{Social skills and information and communications technology as factors associated with the performance of elementary school students affected by forced displacement}

\author{
Inírida Avendaño-Villa \\ Omar Fernando Cortés-Peña** \\ Hilda Guerrero-Cuentas \\ Universidad de la Costa, Colombia
}

Recibido: 20 de octubre de 2014 Revisado: 30 de noviembre de 2014 Aceptado: 12 de diciembre de 2014

\section{Resumen}

El presente artículo tiene como propósito central describir los principales referentes teóricos y hallazgos derivados del análisis relacional entre las competencias sociales, las actitudes frente al uso de las TIC y el desempeño académico a partir de los reportes de una muestra conformada por docentes (9), padres de familia (60) y estudiantes (60) del nivel de básica primaria en la I.E. Villa Campo (Malambo) e I.E.D. Carrizal (Barranquilla), quienes han sido expuestos a la experiencia del desplazamiento forzado. Para tal efecto, se administró inicialmente el protocolo de consentimiento informado y a cada uno de los diferentes actores se les administró según su correspondencia los instrumentos de Actitudes frente al uso de las TIC, Cuestionario de Solución de Conflictos Escolares, Escalas MESSY para Estudiantes y Docentes, Inventario de Observación de Conductas Escolares, Cuestionario de Caracterización Sociodemográfica y Percepción sobre el uso de las TIC para los padres de familia. Los resultados reflejan una actitud positiva frente al uso de las TIC en los estudiantes y docentes, el empleo de estrategias de solución de conflictos ante la prevalencia de exposición a eventos de agresión escolar, un mayor nivel

Artículo de investigación. Proyecto INDEX CONV-08-2013: Competencias sociales, tecnologías de la información y la comunicación y desempeño académico en estudiantes con experiencia de desplazamiento forzado.

Correspondencia: Omar Fernando Cortés Peña Correo, ORCID iD http://orcid.org/0000-0002-9603-885X, Institución Corporación Universidad de la Costa (CUC), Colombia. Dirección: calle 58 \# 55-66, Facultad de Psicología, Corporación Universidad de la Costa (CUC), Barranquilla, Colombia. Correo electrónico: ocortes3@cuc.edu.co

Inírida Avendaño: http://orcid.org/0000-0003-3390-4797

Hilda Guerrero: http: // orcid.org/0000-0002-8130-7519 
de reconocimiento de conductas Prosociales en comparación con el reporte de conductas agresivas por parte de los docentes y el establecimiento de asociaciones estadísticamente significativas entre las competencias sociales de conducta prosocial y solución de conflictos de forma directamente proporcional, a diferencia de la relación inversa entre la conducta agresiva y la conducta prosocial; así como una relación directamente proporcional entre las conductas Prosociales y el desempeño académico y una asociación estadísticamente significativa entre el uso de TIC y el desempeño académico.

Palabras clave: desplazamiento forzado, competencias sociales, TIC, desempeño académico, educación básica primaria.

\section{Abstract}

The main goal of this paper is to describe the main theoretical framework and findings from the relational analysis of social skills, attitudes to ICT and academic performance reports from a sample made up of teachers $(n=9)$, parents $(n=60)$ and students $(n=60)$ in two elementary schools in the cities of Malambo and Barranquilla, Colombia, who have experienced forced displacement. To this end, a protocol consisting of an informed consent form, the Attitudes towards ICT Questionnaire, the School Conflict Settlement Scales, the MESSY scale for Students and Teachers, a School Behavior Observation Inventory, a Sociodemographic Characterization Questionnaire and a Perception on the use of ICT for parents scale. Results reflect a positive attitude towards the use of ICT on students and teachers, the use of conflict resolution strategies related to exposure to bullying, a higher level of recognition of prosocial behaviors compared with the report on aggressive behavior on the part of teachers and the establishment of statistically significant associations between social skills and prosocial conflict resolution in direct proportion, unlike the inverse relationship between aggressive behavior and prosocial behavior, as well as a directly relationship proportional between prosocial behavior and academic performance and a statistically significant association between the use of ICT and academic performance .

Keywords: Forced displacement, social skills, ICT, academic performance, basic primary education

\section{Introducción}

El presente artículo parte de la necesidad de analizar el papel que tienen las competencias sociales y el uso de las tecnologías de la información y la comunicación (TIC) como factores predictores del desempeño académico en estudiantes de básica primaria, con experiencia de desplazamiento forzado en el departamento del Atlántico. Como el proyecto lo expresa, el desplazamiento es una realidad que se ha venido viviendo en el mundo, pero más específicamente en Colombia, entendido de alguna manera como un flagelo que enfrenta este país, a pesar de los programas gubernamentales, de los avances en las propuestas de militarización, y de los rubros económicos destinados a invertir en soluciones para el conflicto. Una de las consecuencias de este es el desplazamiento forzoso, cuyo impacto puede evidenciarse de forma integral en la calidad de vida de las personas al generar un contexto negativo para su desarrollo $y$, por ende, para el de los procesos educativos.

En el mismo sentido, existen factores asociados al desplazamiento, como la pobreza, los cuales generan consecuencias directas sobre el desempeño académico de los estudiantes. Por ejemplo, Ordoñez (2009) expresa que las deficiencias nutricionales, principalmente el déficit en antioxidantes, ácidos grasos omega 3 y 6 , zinc, magnesio y aminoácidos esenciales es un factor que afecta significativamente a los niños y niñas participantes en 
la presente investigación. De igual forma, Amarís (2004), en su estudio Relaciones familiares en familias desplazadas por la violencia ubicadas en "la cangrejera", plantea que la dinámica familiar se modifica en entorno al desplazamiento; llegar a nuevos espacios y lograr obtener un lugar donde vivir permite la recreación visual de sus actuales viviendas como eran las de sus lugares de origen (Amariles, 2008).

Por otra parte, la UNESCO - OREALC (2003) manifiesta que "los principales indicadores para medir el progreso hacia la educación básica universal (EPT) tienen relación con el acceso y la participación (tasas netas de admisión y matricula), así como con la evolución o la terminación (tasas de supervivencia-retención o tasa bruta de admisión en el último año de una etapa)" (Vélaz, 2005, p.3). Colombia no es ajena a esta situación; si bien en los últimos años se han presentado mejoras que la ubican en una buena posición frente a otros países latinoamericanos, las cifras del DANE (2011) sobre el acceso de la población a herramientas como internet indican que aún es muy limitado, especialmente en las zonas rurales del país.

Para 2010, el $26 \%$ de los hogares colombianos poseía computador, de los cuales el $32,5 \%$ se encontraba en las ciudades y áreas metropolitanas, y el $4 \%$, en las zonas rurales. De igual forma el $19,3 \%$ de los hogares poseía conexión a internet, de los cuales el $24,4 \%$ se encontraba en las ciudades, mientras el $1,6 \%$, en el campo. Por otra parte, las instituciones públicas de educación básica, generalmente, cuentan con equipos de segunda, dotados de tecnología obsoleta, de manera que los estudiantes se forman con herramientas desactualizadas, que limitan sus posibilidades de desempeño académico y, posteriormente, laboral (DANE, 2003). "Un ejemplo de esto es que para el año 2003 , el $75 \%$ las escuelas públicas que contaban con equipo de informática para sus estudiantes aún tenían el sistema operativo de Windows 95-98" (DANE, 2003), y "solo el 10\% de estos computadores se utilizan para labores pedagógicas" (DANE, 2003).

El enfoque de esta investigación se centra en el sector educativo de la población de Malambo, donde el $82 \%$ de los estudiantes matriculados en primaria y bachillerato recibe el servicio educativo por parte del sector público (Alcaldía Municipal de Malambo, 2008). Ahora bien, de cada 100 niños en edad escolar, 40 no están asistiendo a una institución educativa formal, lo que indica que la cobertura educativa del municipio es de un $27 \%$, y la permanencia de los niños en el colegio se encuentra bastante afectada, en especial en los sectores más vulnerables (Alcaldía del Municipio de Malambo, 2008).

Así pues, en relación con el análisis del rendimiento escolar, existen una serie de factores determinantes. Gran parte de la investigación sobre estos factores del rendimiento escolar durante los últimos decenios ha destacado la importancia de las características de la familia y los estudiantes (Bloom, Engelhart, Furst, Hill, \& Krathwohl, 1956; Coleman et al., 1966; Hanushek y Luque, 2003; Mejía, 2006; Bravo, 2011; Cubero, 2005; Chaves, 2001).

Las características de los antecedentes familiares, en particular los ingresos y la educación de los padres, y, en menor grado, los recursos educativos en el hogar, han resultado estar asociadas de manera significativa y positiva con el logro escolar. Las características de los alumnos exploradas incluyen rasgos inherentes, tales como el sexo y la edad, así como características que pueden estar más influenciadas por políticas, como la salud y el alfabetismo temprano. Estas últimas han demostrado tener una asociación positiva con el logro escolar y con un impacto a largo plazo sobre aquel. (Banco Mundial, 2008, p. 36).

En el marco internacional, Escobar (2012) presenta, en su estudio adelantado en Nicaragua, las implicaciones derivadas del bajo rendimiento académico de los estudiantes de una comunidad educativa del municipio de Bluefields. En este estudio se resaltó el bajo interés que presentan los estudiantes, asociado con un bajo nivel de participación de los padres y la mínima implementación de estrategias pedagógicas activas por parte de los docentes, aspectos que se encuentran coyunturalmente unidos al déficit de dotación de recursos y materiales de apoyo bibliográfico y pedagó- 
gico, lo que a su vez permite ver las dificultades y falencias asociadas con los procesos de gestión administrativa.

Uno de los tópicos centrales del presente estudio hace referencia a la necesidad de identificar la complejidad de la realidad a la que están expuestos los niños y niñas con experiencia previa en desplazamiento forzado, y cómo este contexto puede incidir negativamente en su desempeño académico. El fenómeno de desplazamiento forzado marca una huella psicosocial, con diversas repercusiones en la población, y afecta además el contexto educativo, dado el índice elevado de niños y niñas con experiencia de desplazamiento forzado.

Si bien es cierto que la problemática del desplazamiento implica un papel activo del Estado en la restitución y reparación integral, también es importante superar las tendencias paternalistas y, en tal sentido, la escuela debe apelar a su esencia, que es la educación como insumo para la transformación a partir de la enseñanza y el desarrollo de nuevos modelos de convivencia social (Amar, Abello, Martínez, Monroy, Cortés y Crespo, 2011).

El efecto que causa en los seres humanos la situación de desplazamiento forzado genera sentimientos de ambivalencia, con contenido de pérdida, en razón del desprendimiento de su contexto, lo que genera en el individuo una necesidad de protección contra la evocación de situaciones amenazadoras, unido a otras consecuencias negativas de orden psicosocial.

La evaluación de los desempeños de los niños desplazados en las escuelas requiere tratamiento diferencial, puesto que las condiciones psicosociales así lo exigen. El Ministerio de Educación Nacional, mediante los programas enfocados en la escuela y el desplazamiento, trata de mitigar las disfunciones que presentan niñas y niños en condición de desplazamiento y reingresarlas al sistema en el nivel correspondiente.

La adaptación al nuevo territorio no privilegia el acceso a las instituciones escolares; por lo general, se ocasionan problemas de desintegración fa- miliar, extra edad, deserción, bajo nivel de aprendizaje y bloqueo emocional. El sistema educativo debe procurar adaptarse a las condiciones de los niños desplazados, para lo cual debe contar con recursos y programas de investigación y análisis de realidad, además de claras políticas de formación de docentes para enfrentar las vicisitudes propias del desplazamiento, que impliquen el reconocimiento de la dignidad humana de todos los ciudadanos. La formación de maestros para el tratamiento de problemas de desplazamiento y todas las consecuencias psicopedagógicas que este fenómeno acarrea requieren un trato especial. (Correa de Molina, 2004; Correa, 1999; Delors et al., 1996; Freire, 1969 y Sabater, 1997).

\section{Método}

El abordaje metodológico es cuantitativo y está sustentado en el uso de escalas de medición de las competencias sociales, el inventario del uso de las TIC y los indicadores objetivos del reporte de desempeño académico.

\section{Tipo de estudio}

Para el desarrollo del presente estudio se asumió el modelo empírico-analítico, con un diseño no experimental transversal de dos grupos, que incorpora los niveles de análisis de orden descriptivo, comparativo, correlacional y predictivo. Las variables centrales del estudio a escala predictora fueron: uso de las TIC en los procesos de enseñanza; actitud frente al papel de las TIC en el aprendizaje; competencias sociales positivas -conductas prosociales-; competencias sociales negativas -conductas agresivas-; competencias sociales de negociación -solución de conflictos escolares-; competencias sociales -observación de conducta escolar-. La variable central criterio del estudio fue el desempeño escolar.

\section{Muestra}

Estudiantes de básica primaria de las instituciones educativas IE Villa Campo del municipio de Malambo, Atlántico, e IE Distrital María Inmacu- 
lada de Carrizal, sedes 1 y 2 . Las muestras independientes de niños y niñas de las mismas instituciones mencionadas fueron no probabilísticas. En total, 60 estudiantes ( 30 por institución) fueron seleccionados a partir de un muestreo no probabilístico bajo el criterio de sujetos tipo, en el cual la variable central de inclusión se estableció en función de su condición de exposición previa a las circunstancias violentas y eventos adversos del desplazamiento forzado. Los criterios de inclusión fueron los siguientes:

- Edad: 7 a 15 años.

- Género: masculino y femenino.

- Experiencia de desplazamiento forzado: mayor a 6 meses.

- Escolaridad: básica primaria.

\section{Instrumentos}

Los instrumentos empleados en el desarrollo del estudio han sido ampliamente aplicados e incorporados en diferentes investigaciones relacionadas con el desarrollo de las competencias sociales y el análisis de las conductas agresivas y prosociales en el contexto escolar (Matson, Macklin y Helsel, 1985; Matson y Ollendick, 1988; Matson, Rotatori y Helsel, 1983; Trianes, Blanca, Muñoz, García, Cardelle-Elawar e Infante, 2002; Trianes y Muñoz, 1989; Trianes, Cardelle-Elawar y Blanca, 2001; Trianes, Muñoz y Jiménez, 1997; Trianes, De la Morena y Sánchez, 1996).

- Escalas MESSY, versiones estudiante profesor: Su nombre original es The Matson Evaluation of Social Skills in Youngsters (MESSY), de Matson, Rotatori y Helsel (1983). La escala MESSY para el estudiante está conformada por 62 ítems; para el profesor, por 64.

- Inventario de observación para profesores: Su nombre original es Escala de Observación para Profesores (EOP), de Muñoz, Trianes, Jiménez, Sánchez y García (1996).

- Cuestionario de solución de conflictos y eventos de agresión escolar: El cuestionario de solución de conflictos y eventos de agresión escolar fue presentado por Ortega y Del Rey $\square 2003$ en su estudio sobre la violencia escolar, y conformado por dieciséis ítems, con una escala ordinal de frecuencia (nunca, alguna vez, a veces, muchas veces).

- Cuestionario de actitud frente a las TIC, asociado con las competencias sociales y el desempeño académico para padres de familia: Este cuestionario fue diseñado por los investigadores del presente proyecto (Avendaño, Cortés y Guerrero, 2013); cuenta con una dimensión de información sociodemográfica, una segunda dimensión de preguntas asociadas con el uso de computador y TIC, y finalmente preguntas referidas a las competencias sociales y al desempeño académico de sus hijos.

\section{Procedimiento}

Para el desarrollo del estudio, en primera instancia se solicitó el consentimiento informado de los padres y el consentimiento institucional, tanto en el IED Carrizal, como en el IED Villa Campo. Posteriormente se desarrolló una sesión de socialización y sensibilización con los estudiantes; después se adelantó una sesión de administración de los instrumentos, los cuales fueron procesados con el software SPSS V. 18.0. Los resultados fueron socializados en cada una de las dos instituciones educativas, bajo el formato de seminario-taller.

\section{Resultados}

A continuación se presentan los principales hallazgos que se encontraron en los instrumentos aplicados en la investigación, en cumplimiento de los objetivos específicos. En primera instancia se analizan los resultados relacionados con la caracterización del perfil de los docentes, y específicamente su actitud y uso de las TIC en el contexto educativo. En segunda instancia se identifican los principales hallazgos derivados de los reportes que hacen los estudiantes en su caracterización sociodemográfica, su opinión acerca del uso de las TIC en los procesos de aprendizaje y la vida cotidiana, así como sobre sus competencias sociales en las dimensiones de conducta agresiva y conducta prosocial, y estrategias de resolución de 
conflictos. Igualmente se analizan los resultados derivados del reporte que adelantan los profesores sobre las competencias sociales de los estudiantes en las dos dimensiones mencionadas. Se analizan los resultados de los reportes de observación de conducta escolar, así como las características sociodemográficas y la opinión que tienen los padres de familia sobre el papel de las TIC en los procesos de aprendizaje de sus hijos. Finalmente se presentan los principales hallazgos relacionados con las asociaciones existentes entre las competencias sociales (conducta agresiva y conducta prosocial), estrategias de resolución de conflictos, uso de las TIC y nivel de rendimiento académico a partir de los reportes de los profesores, estudiantes y padres de familia.

\section{Caracterización sociodemográfica y uso de las TIC por parte de los docentes}

De los docentes, 55,6\% pertenece a IE Villa Campo, y $44,4 \%$, a la IED Carrizal. El $88,9 \%$ son mujeres y el $11 \%$ restante son hombres, con edades entre 27 y 48 años en un $67 \%$. Respecto al estado civil de los encuestados, 55,6\% de los docentes son casados, y $44,4 \%$, solteros; $66,7 \%$ pertenecientes al estrato 3; 22\%, al 4; y $11 \%$, al 2. En cuanto al nivel de sus estudios de pregrado, se resaltan las áreas de psicología, educación física, matemáticas y ciencias sociales. El promedio de experiencia de los docentes es de 15 años, y el

Figura 1. Actitud frente al uso de las TIC en los docentes

TIC: Fortalece el desarrollo de competencias sociales

TIC: Promoción de nuevos escenarios de enseñanza

TIC: Herramientas complejas en procesos de enseñanza

TIC: Motivación con las experiencias de aprendizaje

TIC: Favorece competencias sociales

TIC: Favorece desempeño académico

TIC: Promueve fortalecimiento de modelos pedagógicos

TIC: Limita desarrollo de procesos pedagógicos

TIC: Apoyan mejoramiento continuo docente

TIC: Medio de acceso restringido
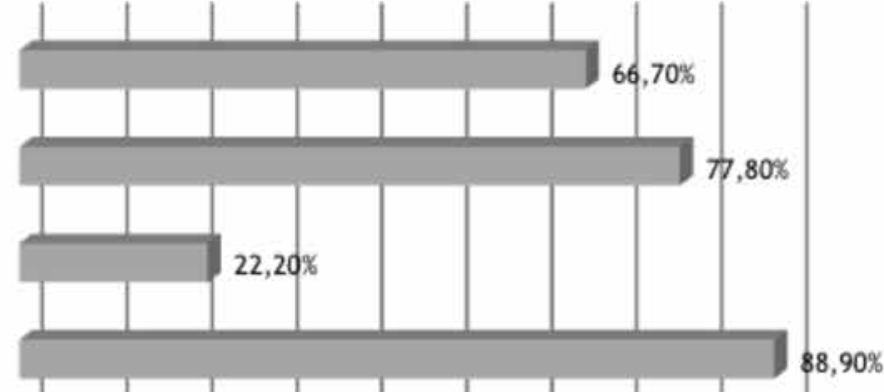

$0,00 \% \quad 10,00 \% \quad 20,00 \% \quad 30,00 \% \quad 40,00 \% \quad 50,00 \% 60,00 \% 70,00 \% 80,00 \% 90,00 \%$

Porcentaje moderado y total acuerdo

Fuente: elaboración propia (2014). 
promedio de antigüedad en sus correspondientes instituciones es de 7 años. En relación con el nivel educativo de los docentes, el 55,6\% tiene pregrado, un $33,3 \%$ es especialista, y el $11,1 \%$ es normalista. La figura 1 ilustra el perfil general de actitud de los docentes frente al uso de las TIC.

La figura 2 presenta el perfil asociado con la frecuencia y el uso de TIC en los docentes. En este sentido, el 55,6\% de ellos considera que el uso de
TIC promociona el desarrollo del aprendizaje, y aspectos tales como el uso de TIC en actividades sociales, su papel en los procesos de comunicación entre docentes y estudiantes, su aporte al progreso de la educación, y su rol en el desarrollo de competencias sociales se evidencian en un 33\% de los casos.

La tabla 1 presenta los resultados obtenidos en las escalas actitud docente y uso docente de TIC.

Figura 2. Frecuencia de uso de las TIC en los docentes

Uso de TIC en actividades de competencias sociales

Uso de TIC en evaluaciones del desempeño académico

Direccionamiento pedagógico basado en uso de TIC

Retroalimentación de los estudiantes con uso de TIC

Diseño estrategias motivadoras para uso de TIC

Investigación basada en TIC a nivel educativo

Aplicación de estrategias pedagógicas basadas en TIC

Promoción del diálogo con estudiantes sobre uso de TIC

Promoción del uso de TIC en procesos de aprendizaje

Implementación de actividades sociales con TIC

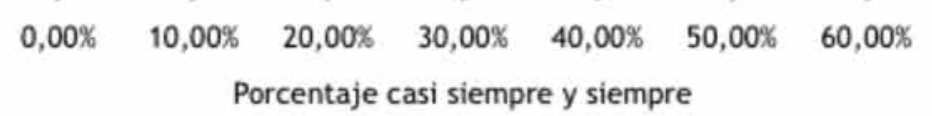

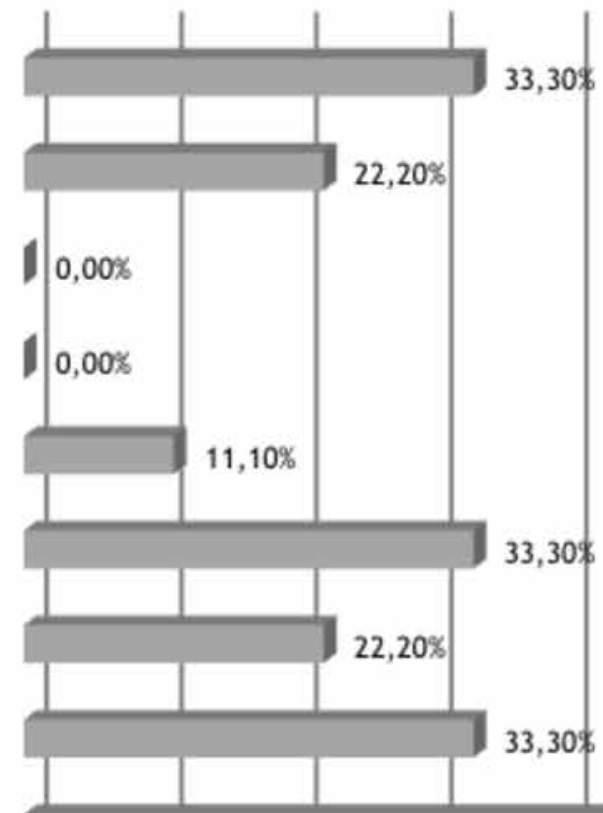

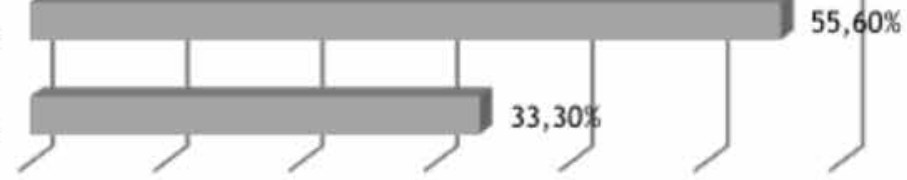

Porcentaje casi siempre y siempre

Fuente: elaboración propia (2014). 
Tabla 1

Análisis de las escalas de actitud y uso docente de TIC

\begin{tabular}{llcc} 
Estadísticos & & & \\
\hline & $\begin{array}{c}\text { Actitud docente sobre las TIC } \\
\text { (escala 1 a 5) }\end{array}$ & $\begin{array}{c}\text { Uso docente de las TIC } \\
\text { (escala 1 a 5) }\end{array}$ \\
\hline $\mathrm{N}$ & Válidos & 9 & 9 \\
Media & Perdidos & 0 & 0 \\
Mediana & 3,9667 & 3,1000 \\
Moda & 4,4000 & 3,2000 \\
Desviación típica. & 4,70 & $2,50^{\mathrm{a}}$ \\
Mínimo &, 80623 &, 42131 \\
Máximo & 2,60 & 2,50 \\
& 25 & 4,70 & 3,70 \\
Percentiles & 50 & 3,1500 & 2,6500 \\
& 75 & 4,4000 & 3,2000 \\
\hline
\end{tabular}

a. Existen varias modas. Se mostrará el menor de los valores.

Fuente: elaboración propia (2014).

Tal como se aprecia en la tabla 1, las dos escalas tienen rangos teóricos de 1 a 5 y se identifica que la actitud $(3,96)$ es más favorable que la frecuencia de uso $(3,1)$. Estos resultados indican que existe una imagen positiva de los docentes sobre el papel de las TIC en los procesos educativos. Pero si bien se encontró una asociación positiva ( $r$ : .244), esta correlación es débil en su magnitud.

\section{Caracterización sociodemográfica de los estudiantes y análisis de los reportes sobre el uso de las TIC y competencias sociales}

A continuación se presentan los principales hallazgos de los reportes suministrados por los estudiantes en relación con su actitud en el uso de TIC y sus competencias sociales, a través de sus estrategias de solución de conflicto, indicadores de conducta agresiva e indicadores de conducta prosocial.

En la muestra se presenta una distribución de grados de escolaridad y género así: su mayor tendencia se presenta en el cuarto grado $(55,9 \%)$, seguida por quinto (23,3\%), y en menor medida segundo (13,3\%), principalmente. En su mayoría, la muestra está conformada por mujeres $(76,7 \%)$, y en su minoría, por hombres $(23,6 \%)$.

La tendencia central en la edad de los estudiantes es de 12 años (31,7\%); las edades oscilan entre 9 y 12 años, principalmente, y entre 13 y 16 años, una minoría. Un aspecto crítico por tener en cuenta en el estudio corresponde a la configuración de los hogares a los cuales pertenecen los estudiantes de la muestra, dadas las condiciones particulares de exposición al desplazamiento forzoso, entre otros factores. Se evidencia que solo un $70 \%$ convive con su madre; un $41,7 \%$, con su padre; y un $71,7 \%$, con sus hermanos. En tal sentido, el porcentaje de hogares nucleares es bajo, $y$, en consecuencia, más de la mitad de los estudiantes solo cuentan con algunas de sus figuras parentales. A continuación, la tabla 2 presenta las principales tendencias relacionadas con la actitud y uso de TIC en los estudiantes.

Según la tabla 2, el $35 \%$ de los estudiantes tiene computador, el $33 \%$ tiene internet, al $83 \%$ le gusta entrar y navegar por internet, el $75 \%$ le gusta conocer amigos por internet, el $90 \%$ resalta su 
Tabla 2

Actitud y uso de las TIC en los estudiantes

\begin{tabular}{lccc}
\hline & & Frecuencia & Porcentaje \\
\hline ¿Tiene & Si & 21 & 35,0 \\
computador? & No & 39 & 65,0 \\
& Total & 60 & 100,0 \\
\hline & Si & 20 & 33,3 \\
¿Tiene internet? & No & 40 & 66,7 \\
& Total & 60 & 100,0 \\
\hline ¿Le gusta entrar & Si & 50 & 83,3 \\
y navegar en & No & 10 & 16,7 \\
internet? & Total & 60 & 100,0 \\
\hline ¿Le gusta & Si & 45 & 75,0 \\
conocer amigos & No & 15 & 25,0 \\
por internet? & Total & 60 & 100,0 \\
\hline ¿Le gustan las & Si & 54 & 90,0 \\
actividades & No & 6 & 10,0 \\
del colegio con & Total & 60 & 100,0 \\
internet? & Si & 50 & 83,3 \\
\hline ¿Cree que el uso \\
del computador
\end{tabular}

Fuente: elaboración propia (2014).

preferencia por las actividades del colegio a través de internet y el $83 \%$ considera que el uso del computador le ayuda en los procesos del colegio. Respecto a las páginas favoritas, los videojuegos ocupan el $43,8 \%$ y Facebook, el 30,8\%, entre otros. El $90 \%$ de los estudiantes reporta tener conocimiento sobre el uso de redes sociales.

La tendencia en horas de navegación reportada por un $70 \%$ de los estudiantes oscila principalmente entre 1 y 3 horas. Por su parte, un 15\% reporta un no uso de internet, y el $15 \%$ restante, entre 3 y 6 horas de uso. En lo referente a las preferencias del uso del computador en casa, el $40,7 \%$ de los estudiantes prefiere utilizar el computador solo, $y$, en menor medida, con los padres, hermanos y amigos. El $89 \%$ de los estudiantes utiliza internet como medio para hacer las tareas.

De otra parte, se resalta que el $70 \%$ de los estudiantes prefiere interactuar personalmente con sus amigos, en lugar de hacerlo por el internet. Este hallazgo tiene una implicación significativa en el uso balanceado de internet y las estrategias que puede brindar la institución para promover el correcto uso de este medio, sin que se convierta en un sustituto del contacto personal.

\section{Escala de solución de conflictos y eventos de agresión escolar}

En relación con la escala de solución de conflictos y eventos de agresión escolar, se reportó un índice alfa de Cronbach (a:.79). La reacción general de los estudiantes ante la imposición de los compañeros principalmente se asocia con la opción de insistir ante ellos para explicar sus ideas en un $45 \%$ de los casos.

A continuación se ilustran las principales tendencias relacionadas con la resolución de conflictos. Tal como se muestra en la figura 3, los ítems resaltados en color verde corresponden a estrategias positivas, mientras que los ítems resaltados en azul corresponden a la evidencia de diferentes formas de conflicto. Dentro de los ítems de estrategias de resolución positiva, se resalta principalmente la alternativa que tienen los estudiantes de buscar a alguien para resolver los conflictos $(2,67)$.

Por su parte, dentro de las evidencias de conflicto, se resaltan en primer lugar los insultos, sobrenombres y agresiones verbales $(2,38)$, y en segundo lugar, los reportes de referenciación negativa (hablar mal a espaldas del otro), con un promedio de 2,18. Cabe anotar que dentro de las principales estrategias de resolución reportadas por los estudiantes, se reporta el diálogo $(63,9 \%)$, seguido por la evitación de peleas $(16,7 \%)$ y el juego $(8,3 \%)$, entre otros.

El 61,9\% de los estudiantes considera que la resolución de los conflictos debe hacerse de manera conjunta entre los profesores y los estudiantes, mientras que el $33,3 \%$ cree que son solamente los profesores quienes deben resolverlos, y un $4,8 \%$ considera que son los estudiantes los responsables de dicha acción. Cabe resaltar que dentro de las principales modalidades registradas como conflic- 
Figura 3. Perfil de puntuaciones promedio en escala (1 a 4): Cuestionario de Solución de Conflictos

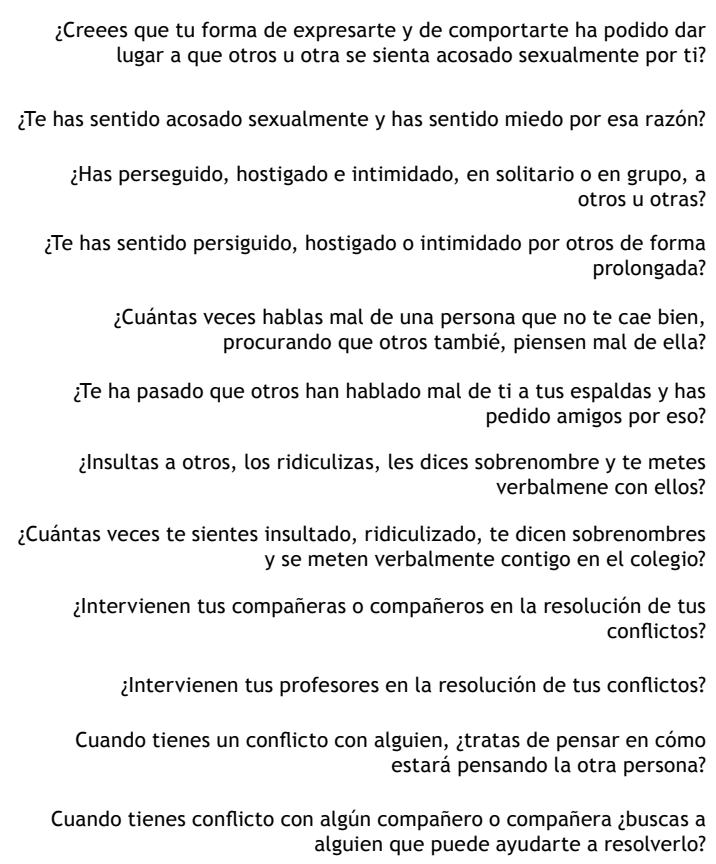

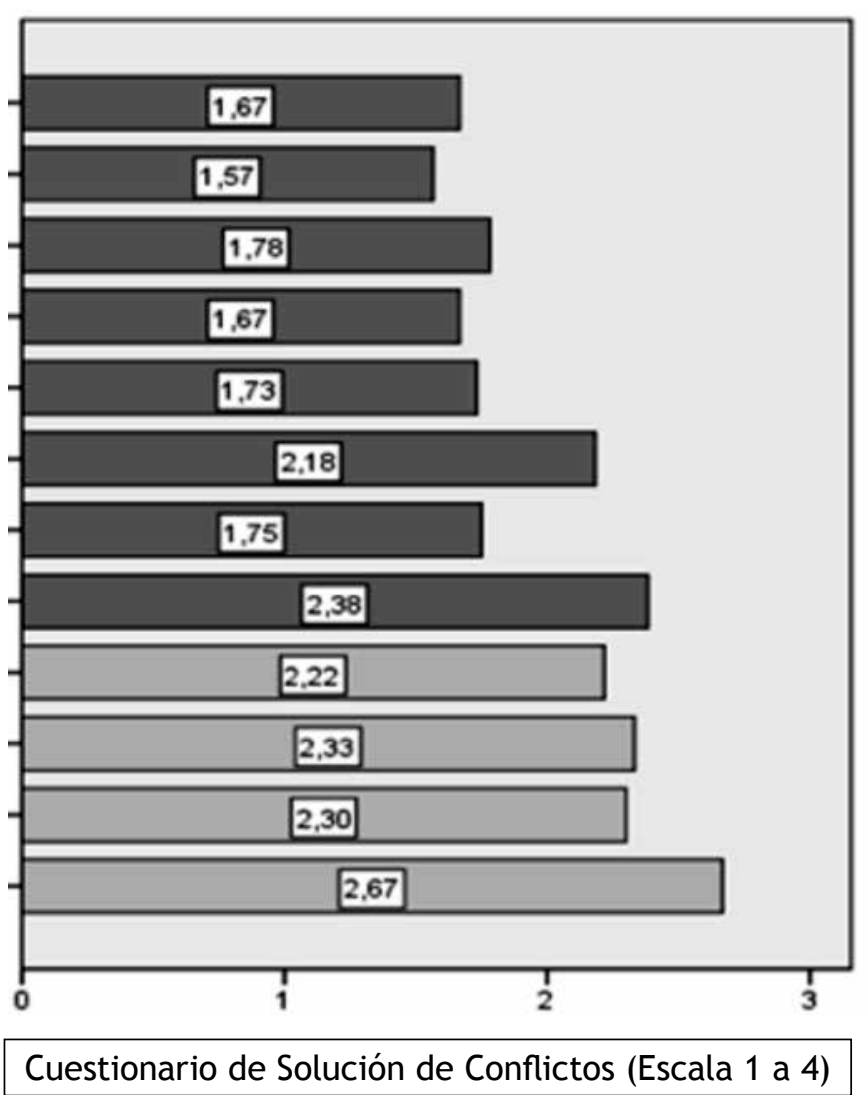

Fuente: elaboración propia

to, se evidencia la agresión física $(40 \%)$ conductas asociadas con acoso sexual (23,6\%), intimidación $(13,3 \%)$ y agresión verbal $(11,7 \%)$.

El puntaje general de la escala de la resolución de conflictos oscila entre 1 y 4 , siendo 1 el de menor nivel de resolución y 4 el de mayor. En particular, el promedio obtenido en el estudio fue de 2,9, lo cual indica una tendencia positiva frente al uso de estrategias de resolución, independientemente de la prevalencia de exposición a los conflictos.

\section{Resultados de la escala MESSY del estudiante}

La escala MESSY de los estudiantes permite medir las competencias sociales en términos de las dimensiones de la conducta agresiva y la conducta prosocial, a partir del autoreporte de los estudiantes en 62 ítems, con una escala de 4 grados de frecuencia, donde 1 es nunca y 4 es siempre. El nivel de confiabilidad de la escala por el método alfa de Cronbach fue de (a: .788).

Dentro de los ítems asociados con la conducta agresiva en los estudiantes, se identificó una tendencia de promedios, comprendidos entre 1,4 y 2,6 , mientras que los ítems asociados con conductas prosociales en su mayoría oscilaron con promedios entre 2 y 3,2 . Frente a los promedios identificados en la escala MESSY en conducta agresiva y conducta prosocial, para los estudiantes, tal como se puede apreciar, el puntaje promedio de la escala de conducta prosocial $(3,43)$ es sensiblemente superior, en comparación con la conducta agresiva $(1,75)$. Estas distribuciones se ilustran de forma discriminada con las figuras 7 y 8 , que reflejan el comportamiento de las escalas en la IE Villa Campo y en la IED Carrizal, en las cuales se resalta el mantenimiento de la misma tendencia general. 
Figura 4. Escalas MESSY de los estudiantes. Conducta agresiva y prosocial en IED Villa Campo.

\section{Institución Educativa Villa Campo}

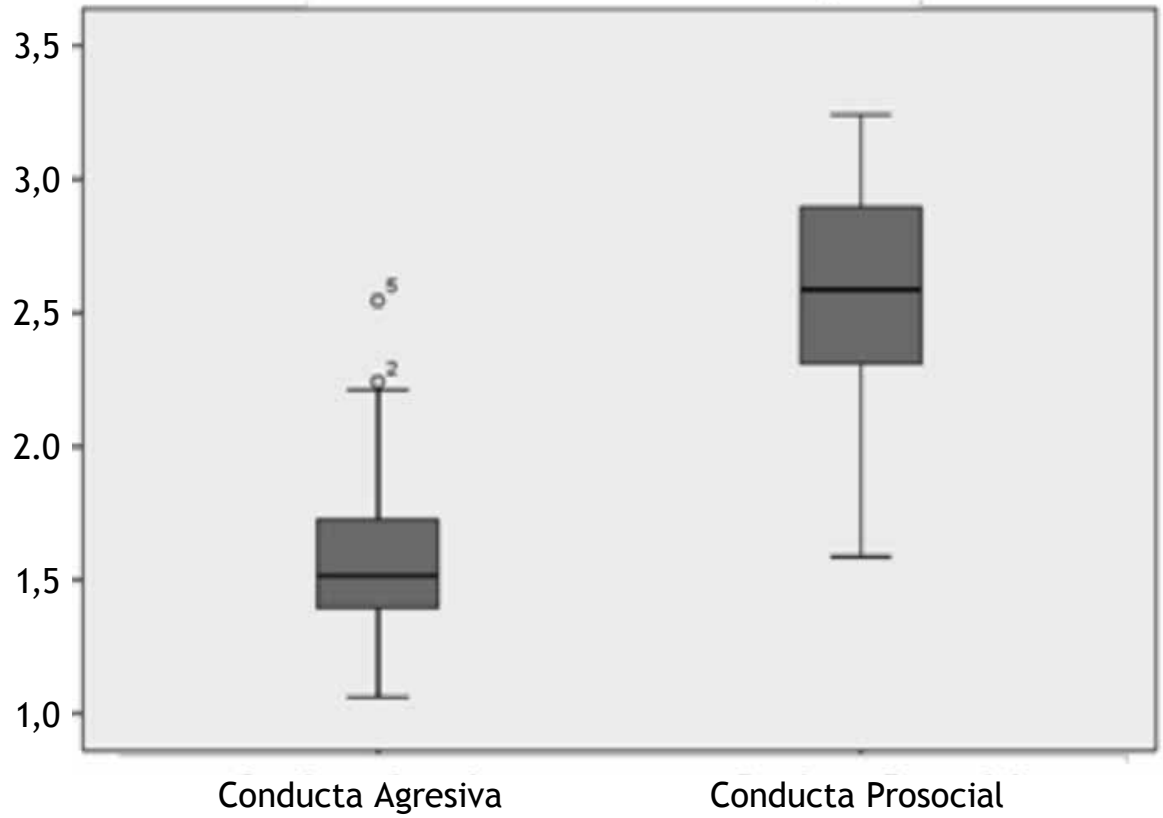

Fuente: elaboración propia (2014).

Figura 5. Escalas MESSY de los estudiantes. Conducta agresiva y prosocial en IED Carrizal.

Institución Educativa Distrital Carrizal

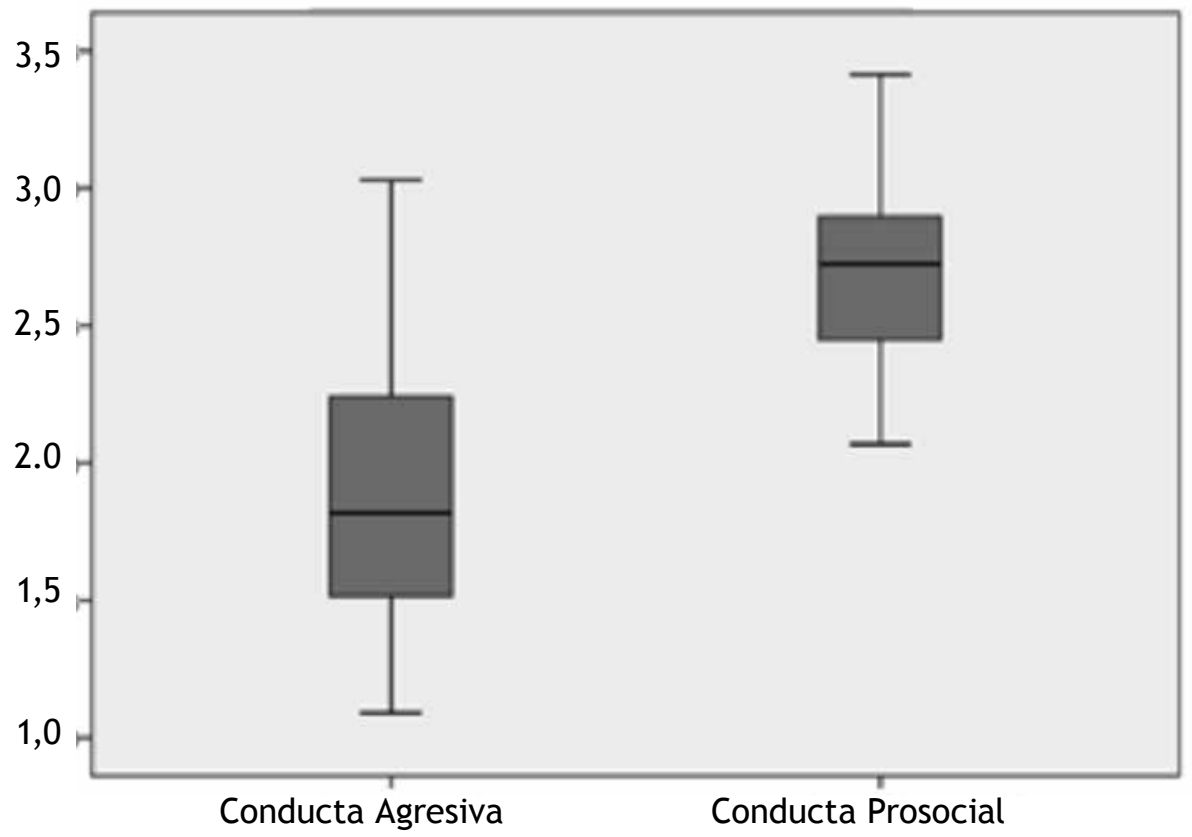

Fuente: elaboración propia (2014). 


\section{Evaluación de las competencias}

\section{sociales de los estudiantes por parte} de los docentes

A continuación, se presentan los resultados centrales derivados del análisis de la escala MESSY, aplicada a los docentes, y de la escala de observación de conducta escolar, reportada por aquellos. En relación con la escala MESSY del docente, se obtuvo un nivel de confiabilidad alfa de Cronbach de (a: .926), el cual es excelente para la muestra. En relación con los ítems de la escala de conducta agresiva, estos oscilan con promedios entre 1,6 y 2,5 , mientras que los relacionados con la conducta prosocial tienden a oscilar entre 2 y 2,9 , tanto al interior de cada institución como a escala consolidada.

En relación con el análisis de la escala MESSY en los docentes, cabe anotar que, nuevamente, la dimensión de conducta prosocial presenta un promedio de conducta superior $(2,45)$, en comparación con los resultados de la escala de conducta agresiva (2.04). Estas tendencias se mantienen al interior de la IE Villa Campo (figura 6), mientras que en la IED Carrizal, los dos niveles de conducta agresiva y prosocial tienden a ser iguales. Estos resultados indican que la percepción que tienen los docentes en Villa Campo tiende a ser más positiva en relación con el desarrollo de las competencias pro sociales reportadas por los docentes en IED Carrizal (figura7).

Por otra parte, los principales hallazgos derivados de la observación de la conducta escolar contaron con una confiabilidad de alfa de Cronbach fue de (a: .893), la cual es alta. Para el caso de la conducta agresiva, los ítems guardaron un alto nivel de homogeneidad, oscilando entre 2 y 2,4, a escala general, y a escala comparada, para el caso de la IE Villa Campo, entre 1,8 y 2,5.Por su parte, en la IED Carrizal oscilaron entre 2,2 y 2,6. Frente al análisis de los ítems relacionados con la conducta prosocial, en general oscilaron por promedios entre 2,1 y 3,1 . En la IE Villa Campo, las variaciones estuvieron comprendidas entre 1,9 y 3,3, mientras que en la IED Carrizal oscilaron entre 3,3 y 2,9 .

Figura 6. Escala MESSY de los docentes en la IE Villa Campo

Institución Educativa Distrital Carrizal

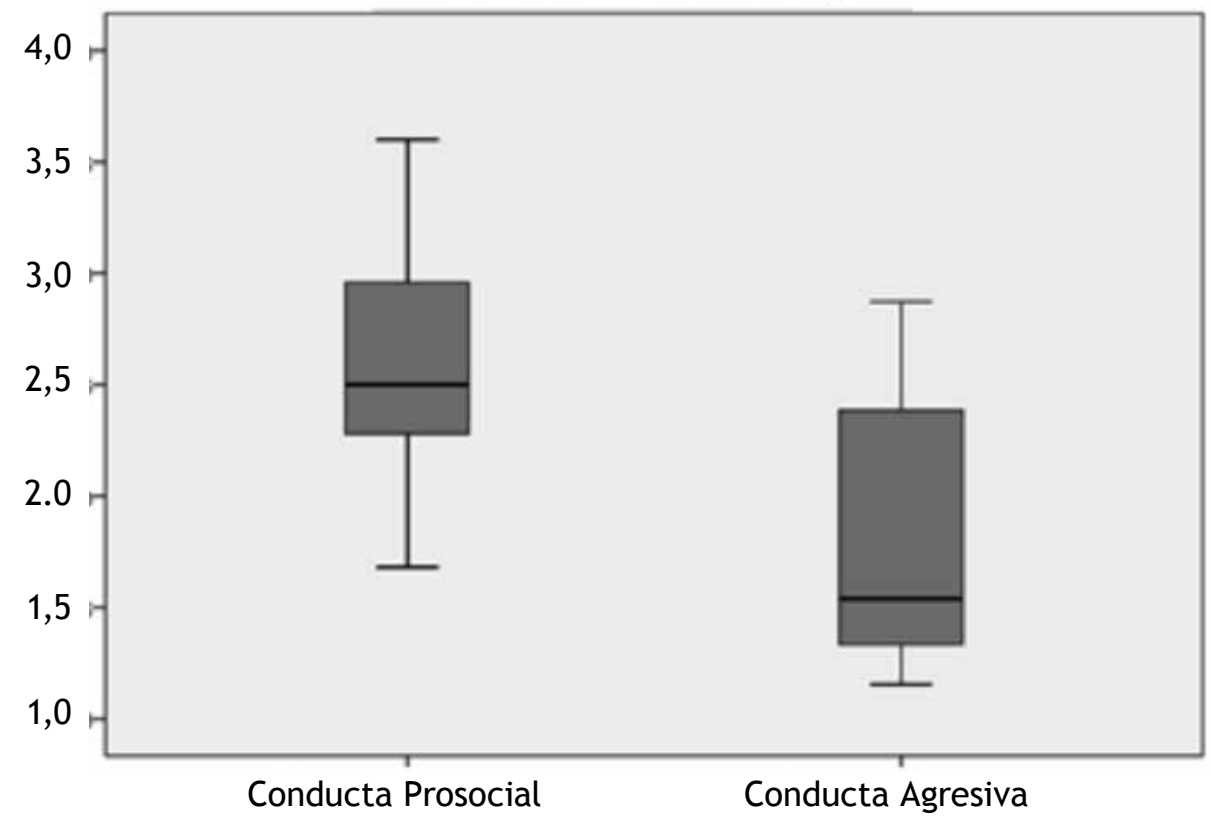

Fuente: elaboración propia (2014). 
Figura 7. Escalas MESSY reportadas por los docentes. Conducta agresiva y prosocial en IED Carrizal.

Institución Educativa Distrital: I.E.D. Carrizal

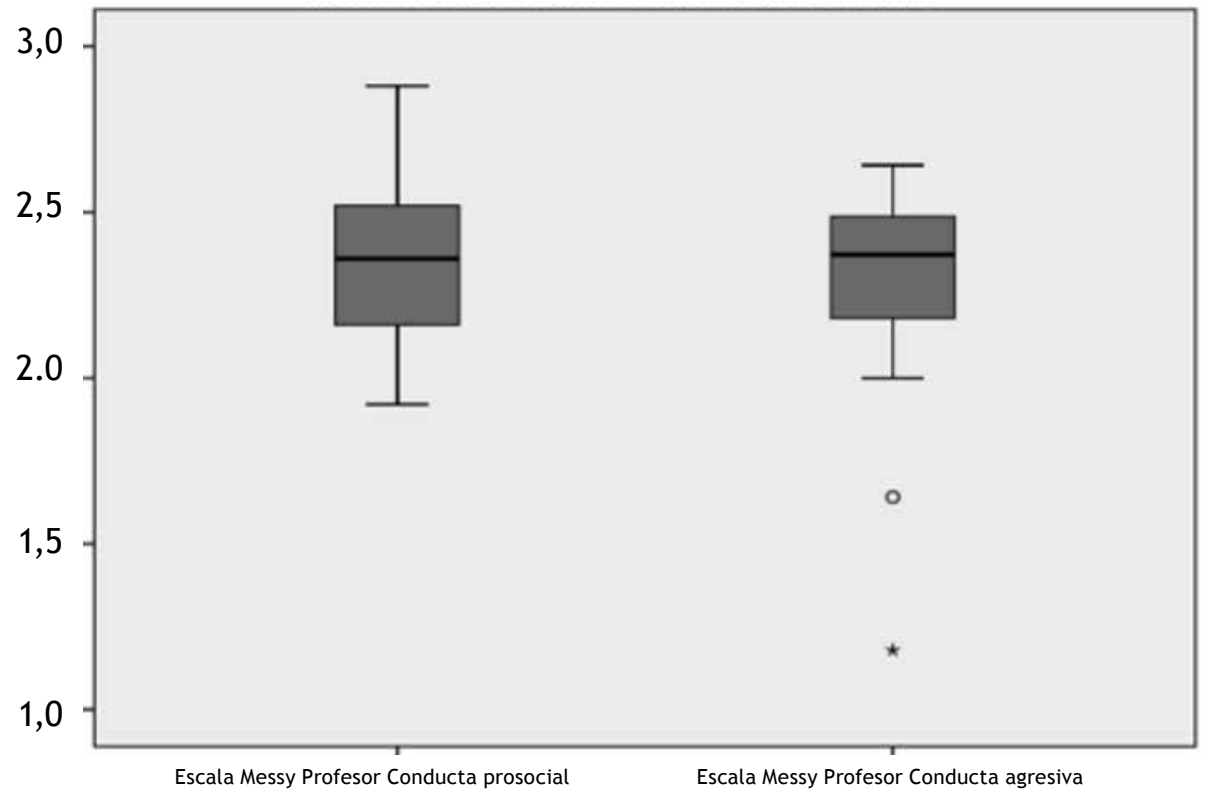

Institución Educativa Distrital Carrizal

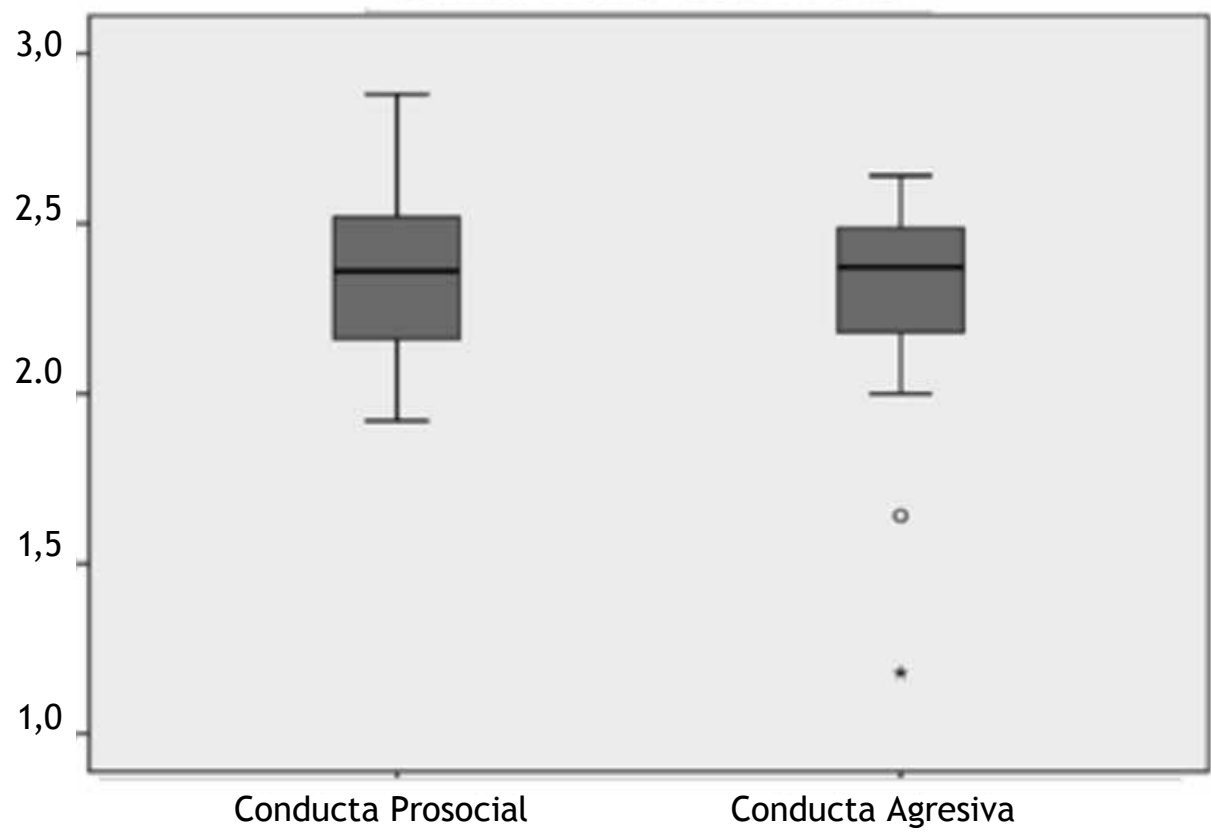

Fuente: elaboración propia (2014).

En términos generales, la tabla 2 presenta el análisis de la observación de las conductas agresiva y prosocial reportadas por parte de los docentes. En este se evidencia cómo el promedio de con- ducta prosocial fue de 2,48, un poco superior al de la conducta agresiva, que fue de 2,26. Estos resultados también se muestran en las figuras 8 y 9. 
Figura 8. Observación de la conducta agresiva y prosocial - IED Villa Campo Institución Educativa Distrital: I. E. Villa Campo

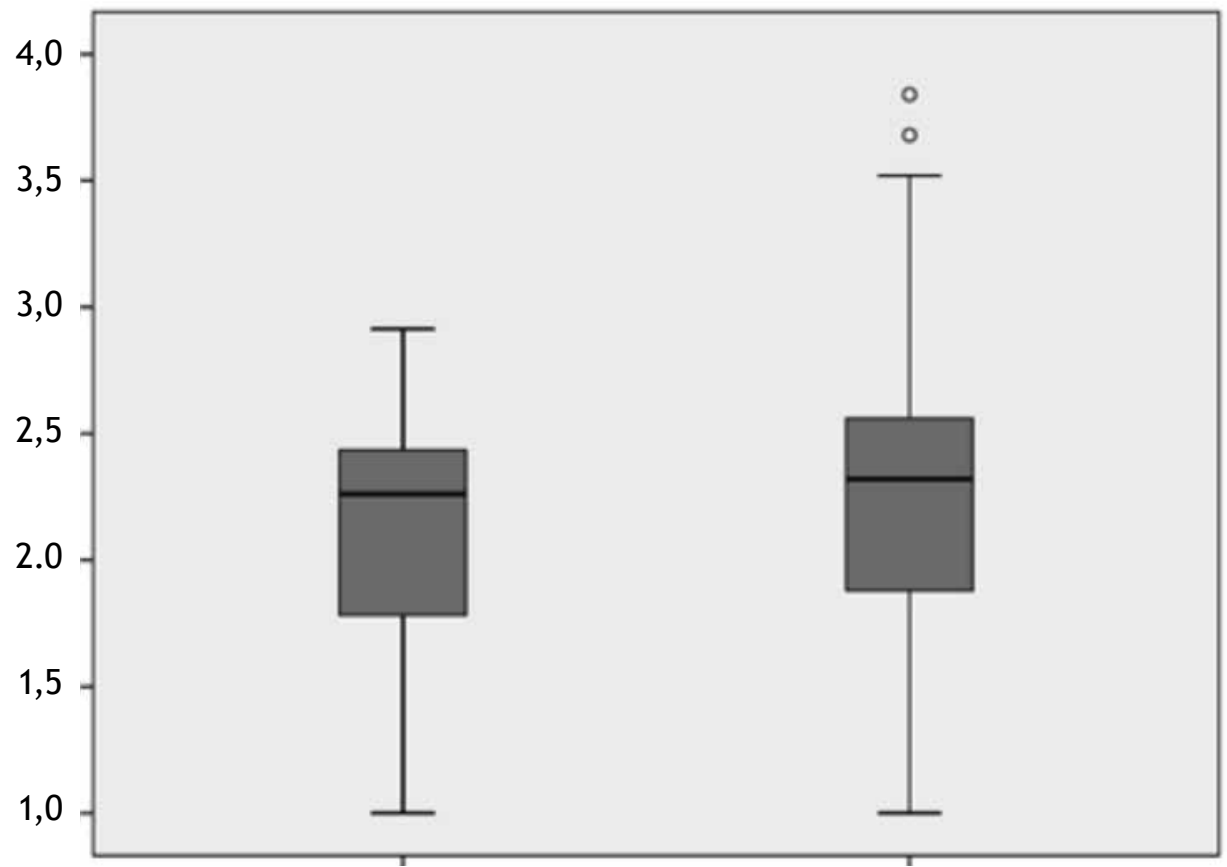

Observación Conducta Agresiva Observación Conducta Prosocial

Institución Educativa Villa Campo

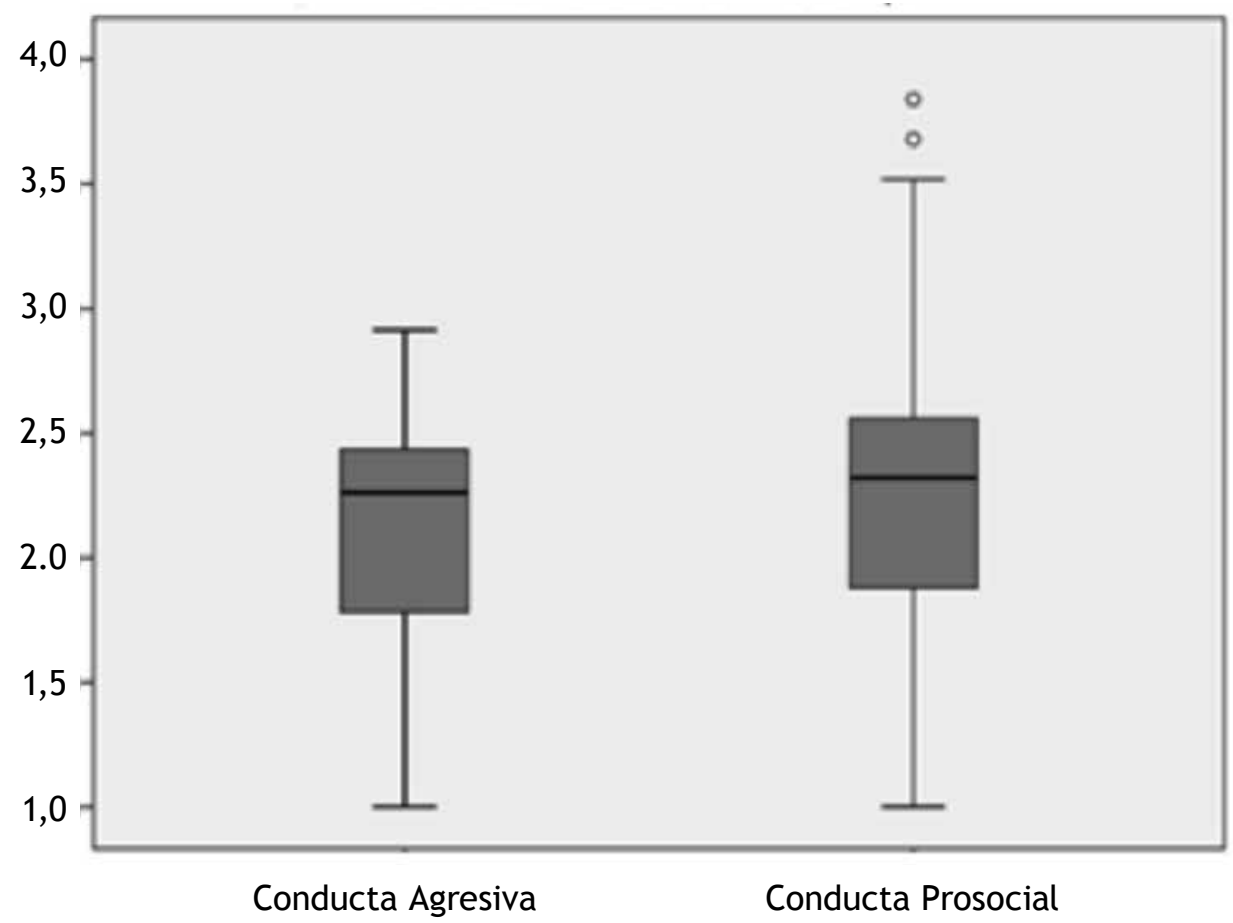

Fuente: Elaboración propia (2014). 
Figura 9. Observación de la conducta agresiva y prosocial en la I E D Carrizal Institución Educativa Distrital: I. E.D. Carrizal

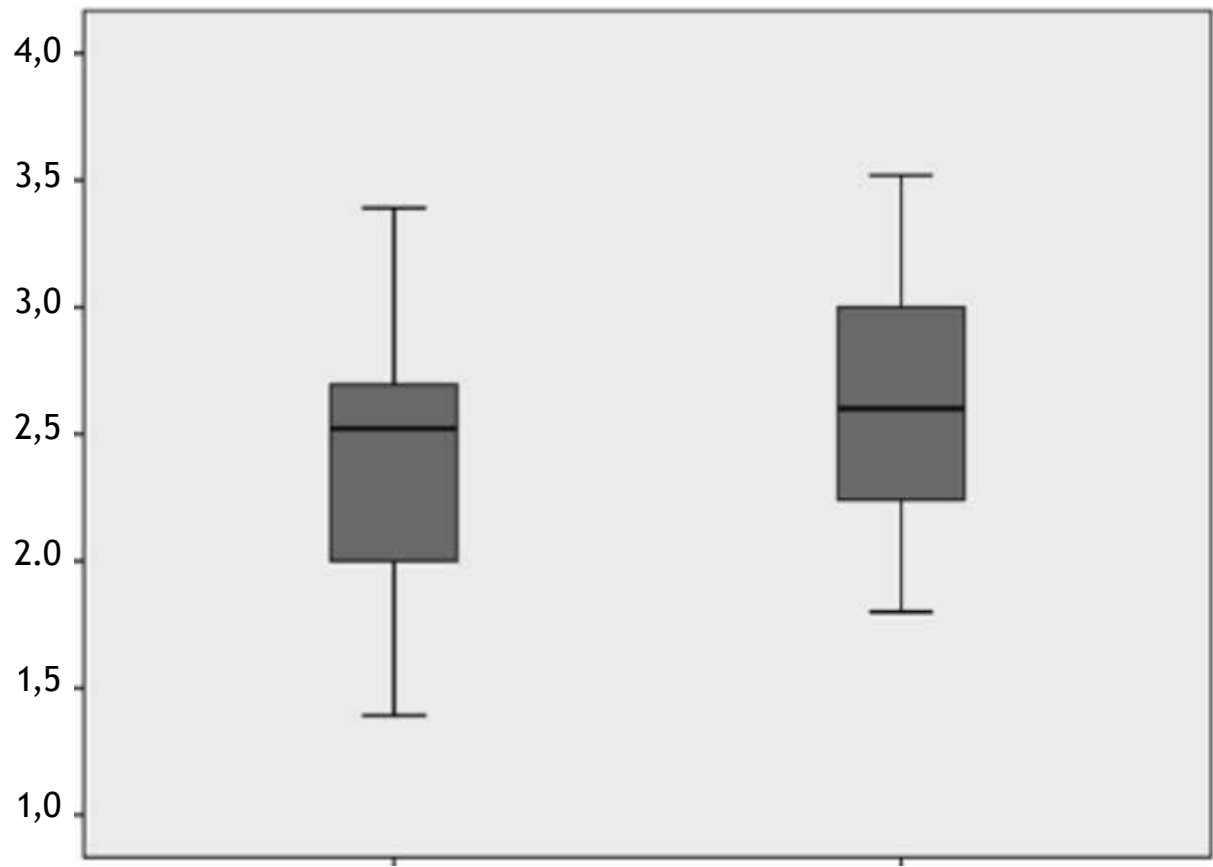

Observación Conducta Agresiva Observación Conducta Prosocial

\section{Institución Educativa Distrital Carrizal}

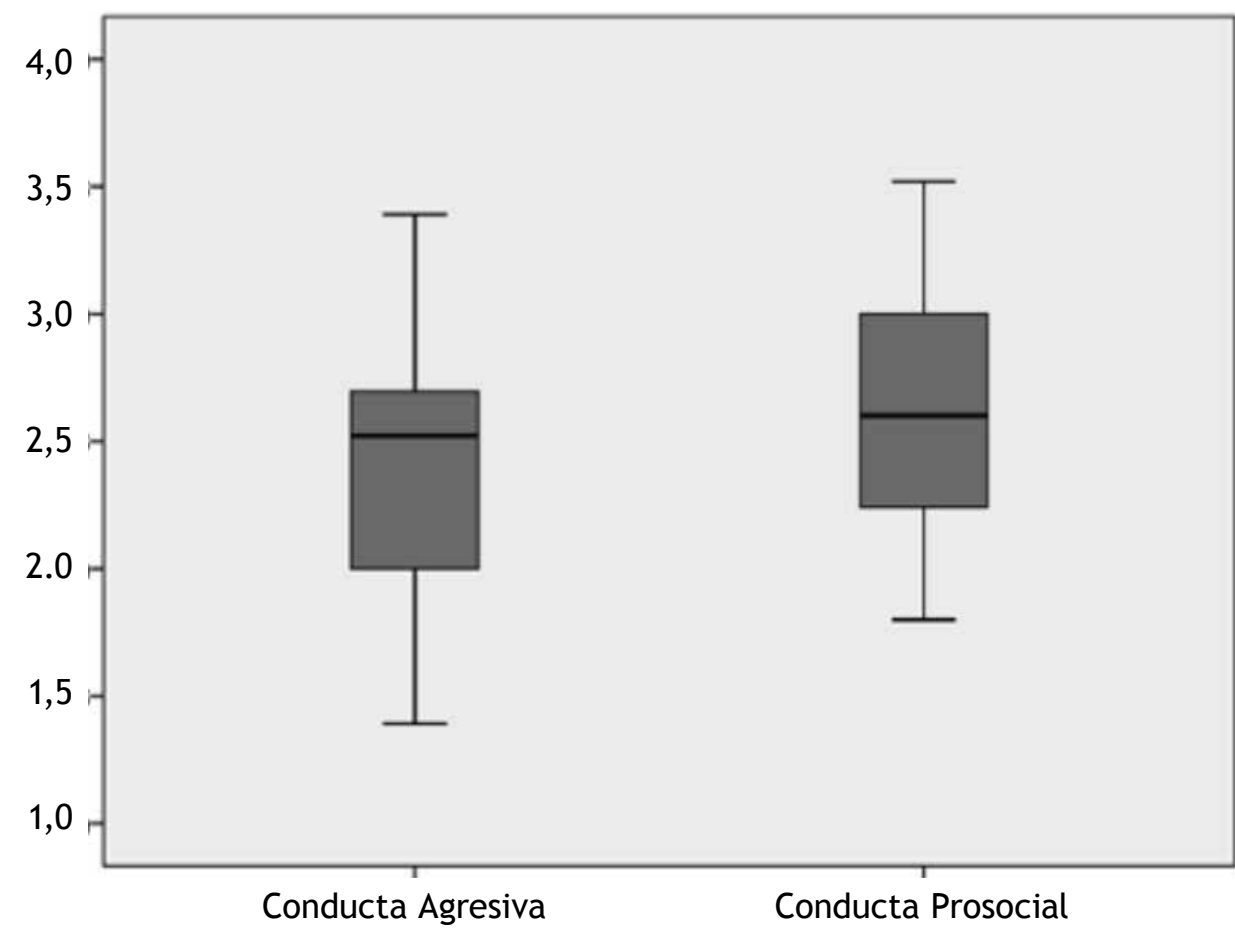

Fuente: elaboración propia (2014). 
Tabla 2

Análisis de la observación de la conducta agresiva y prosocial

\begin{tabular}{lccc}
\multicolumn{4}{c}{ Estadísticos } \\
\hline & $\begin{array}{c}\text { Observación } \\
\text { Conducta } \\
\text { agresiva }\end{array}$ & $\begin{array}{c}\text { Observación } \\
\text { Conducta } \\
\text { prosocial }\end{array}$ \\
\hline $\mathrm{N}$ & Válidos & 59 & 60 \\
Media & Perdidos & 1 & 0 \\
Desviación típica & 2,2638 & 2,4813 \\
Mínimo &, 49467 &, 56203 \\
Máximo & & 1,00 & 1,00 \\
Percentiles & 25 & 3,39 & 3,84 \\
& 50 & 2,9565 & 2,1600 \\
& 75 & 2,6087 & 2,4000 \\
\hline
\end{tabular}

Fuente: elaboración propia (2014).

\section{Percepción del uso de las TIC y su relación con las competencias sociales y el desempeño académico reportados por los padres de familia}

En primera instancia se identifica que el $67 \%$ de las madres y el $32 \%$ de los padres participaron en el reporte sobre las características sociodemográficas en el hogar, y su percepción en el uso de las TIC, las competencias sociales y el desempeño académico reportado por los padres. La distribución de edad de los padres que participaron en el estudio refleja un promedio de 37 años, oscilando principalmente entre los 32 y los 44 años.

En su mayoría, en los padres de familia se reportan las ocupaciones asociadas al hogar (55\%) y al trabajo (43\%), principalmente. Frente al nivel educativo, se evidencia que un $46,6 \%$ de los padres solo cuenta con nivel de formación primaria, y un $31 \%$, con secundaria, lo cual indica por complemento que solo un $23 \%$ ha tenida acceso a algún nivel de formación superior.

En relación con el estado civil de los padres, la unión libre ocupa el primer lugar, con un $45 \%$ de los casos; seguida por los casados, en $26,7 \%$; y los padres separados, en un $13,2 \%$, entre otros. Las tendencias en el número de hijos en el hogar os- cilan entre 1 y 8 . El $63 \%$ de los casos tiene entre 2 y 4 hijos.

Adicionalmente, el $40,35 \%$ de los estudiantes que hacen parte de la muestra del proyecto tienden a ser los menores en sus hogares; el 31,38\%, hijos intermedios; y el $27,0 \%$, los mayores.

Un aspecto por resaltar y tener en cuenta para el desarrollo de futuras intervenciones psico-educativas corresponde al reporte de un $36 \%$ de los casos, en los cuales los padres sospechan alguna dificultad de aprendizaje por parte de sus hijos. Un $40 \%$ de los padres considera que sus hijos son tímidos, mientras que un $30 \%$ reporta que son sociables, y solamente un $18 \%$ da cuenta de ellos como muy sociables.

Frente a los patrones de uso de las TIC, el $48,3 \%$ de los padres y madres reporta el uso de internet, y adicionalmente se evidencia que solo un $30 \%$ acostumbra a navegar con sus hijos. En promedio, los padres dedican a internet 3 horas al día; sus páginas favoritas (Facebook, Google y otras páginas sociales) suman un $73 \%$ de los casos, y sus temas favoritos son las noticias, los video juegos $y$ los contenidos de Facebook. Se observa de forma crítica que solamente el $15 \%$ monitorea o controla el acceso a internet de sus hijos. El $71,6 \%$ considera que el colegio promueve el uso del computador para mejorar el desempeño de sus hijos, lo cual es un referente empírico de la importancia del acceso tecnológico en los procesos de aprendizaje, el cual es resaltado adicionalmente por el $65 \%$ de los padres.

Dentro de las principales razones que argumentan los padres en la relación existente entre el uso de las TIC y el desarrollo social de sus hijos, un $53 \%$ considera que este es un medio que promueve el desarrollo de lazos de amistad. Finalmente las principales ventajas que reportan los padres sobre el uso de las TIC se asocian con el hecho de que consideran su papel facilitador en el desarrollo de las tareas $(52,1 \%)$, su uso para el mejoramiento para los procesos de aprendizaje (33,2\%), y su empleo como facilitador de las relaciones de amistad (12,5\%).

En contraposición, dentro de los principales problemas asociados con el uso de TIC se resalta el 
acceso a páginas inadecuadas $(30,8 \%)$, el uso durante largos periodos de tiempo, dejando de lado otras actividades (25\%), y la vulnerabilidad a ser engañado por internet $(21,2 \%)$.

\section{Análisis correlacional entre el uso de las TIC, las competencias sociales y el desempeño académico}

La figura 10 ilustra la distribución de los niveles de desempeño académico de los estudiantes. Esta distribución asume un comportamiento con tendencia normal, y se resalta principalmente el nivel de desempeño básico en un $40 \%$ de los casos, seguido por el desempeño alto, en un $23 \%$, y el medio, en un $20 \%$. En menor medida se encuentra el nivel superior, en un $10 \%$, y el bajo, en un $6 \%$.

Dentro de las principales correlaciones identificadas, entre las variables de estudio se resaltan las siguientes:
- Escala MESSY del estudiante, conducta agresiva, y escala MESSY del profesor, conducta prosocial. (r:-.49; sig. ,001), denotando una relación inversamente proporcional.

- Escala MESSY del estudiante, conducta agresiva, y escala MESSY del profesor, conducta agresiva. ( $r$ : ,445; sig. ,000), denotando una correlación directamente proporcional.

- Escala MESSY del profesor, conducta prosocial, y escala MESSY del estudiante, conducta prosocial. (r: .382; sig. ,032), denotando una relación directamente proporcional.

- Escala MESSY del profesor, conducta agresiva, y escala MESSY del profesor, conducta prosocial. ( $r$ :-.466; sig. ,000), denotando una relación inversamente proporcional.

- Escala MESSY del profesor, conducta agresiva, y escala de observación, conducta agresiva. $(r$ : .364; sig. ,041), denotando una relación directamente proporcional.

Figura 10. Desempeño académico de los estudiantes

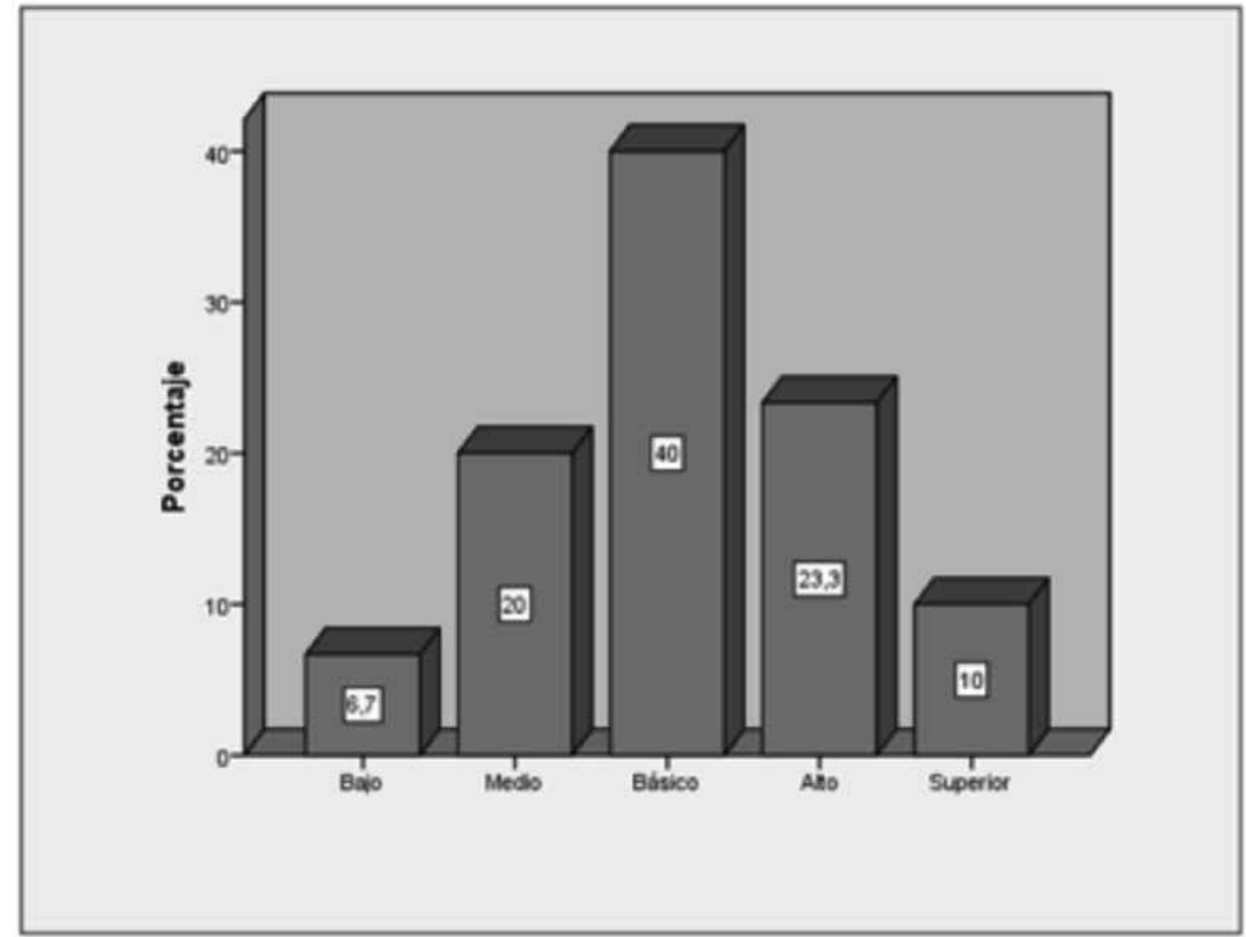

Fuente: elaboración propia (2014). 
- Escala de observación conducta prosocial y desempeño académico ( $r$ :.353; sig. ,044), denotando una correlación directamente proporcional.

- Preferencia por el internet y preferencias por las actividades del colegio a través de internet $\left(X^{2} 5\right.$, 33; sig. 021), en la cual el $80 \%$ de los estudiantes que tiene preferencia por la tecnología se siente motivado por las actividades en el colegio en las cuales se usa internet.

- Desempeño académico y uso de internet como medio para hacer tareas en los estudiantes $\left(X^{2} 4,83\right.$; sig. 4.03), denotando una mayor tendencia al uso de TIC, asociada a los niveles de desempeño alto y superior.

- Desempeño académico y creencia en el uso de TIC como herramienta de ayuda en los procesos de aprendizaje. $\left(X^{2} 12,45 ;\right.$ sig. ,014), denotando una mayor tendencia en el nivel alto $y$ superior.

\section{Discusión}

En el marco general de las conclusiones de esta investigación, a continuación se presentan los principales aspectos sintéticos asociados con los aprendizajes de orden conceptual metodológico empírico y crítico social, como insumo para la promoción de espacios de reflexión del acto educativo, en su constante transformación orientada al aseguramiento de la calidad educativa -que debe ir unida a la calidad de vida-, lo cual cobra mayor pertinencia en contextos particulares como el de los estudiantes que han sido víctimas del desplazamiento forzado, en convergencia con los estudios deGresham \& Cavell (1986), Vargas (1994). Restrepo (1999), Briones (1999), Ballesteros, Cortés y Forero (2001), Rodríguez (2007) y Escobar (2012).

En el ámbito de los aprendizajes conceptuales, el primer aspecto por resaltar es la integración de saberes, en particular desde las disciplinas de la psicología y la educación, lo que hizo un factor diferenciador a partir de los marcos de referencia integrados por la naturaleza del equipo de trabajo interdisciplinar. En este sentido, el abordaje de problemáticas tan complejas, como lo son el uso de TIC, las competencias sociales y el desempe- ño académico en un contexto de estudiantes con previa experiencia de desplazamiento forzado, ameritó la búsqueda de un marco conceptual que integrara los diferentes modelos y teorías de la psicología, la educación y los demás ejes temáticos de las ciencias sociales y humanas, expuestos en los principales autores referenciados en el presente proyecto.

Desde el ámbito metodológico se resaltó el desarrollo de un abordaje empírico-analítico, soportado en el uso de instrumentos válidos y confiables, que han sido objeto de diferentes estudios y publicaciones, así como producto del desarrollo de la línea de investigación por parte de algunos de los miembros del equipo de investigadores.

Específicamente se resalta el uso del instrumento de actitudes frente al uso de las TIC, con sus correspondientes versiones para docentes y estudiantes, diseñado por Avendaño \& Martínez (2012); el cuestionario de solución de conflictos y eventos de agresión escolar, presentado por Ortega y Del Rey (2003); el inventario de observación de conducta escolar para profesores, desarrollado por Muñoz, Trianes, Jiménez, Sánchez y García (1996); las escalas MESSY, versiones para estudiantes y profesores, a partir de los trabajos de Matson, Rotatory y Helsel (1983); y el cuestionario de actitud frente al uso de las TIC, asociado con las competencias sociales y el desempeño académico para padres de familia, diseñado por Avendaño, Cortés y Guerrero (2013). Estos instrumentos permitieron establecer una mirada de 360 grados frente al abordaje de la problemática central objeto de estudio, referida al análisis de las competencias sociales, y el uso de las TIC frente al desempeño académico de los estudiantes con experiencia de desplazamiento forzado.

En lo referente a los aprendizajes derivados del análisis de los resultados desde la perspectiva empírico-analítica, en términos generales se puede identificar lo siguiente:

1. Los docentes tienen una actitud favorable frente al uso de las TIC y valoran su papel en la innovación de los escenarios de procesos de enseñanza y aprendizaje, así como su aporte al mejoramiento continuo de su rol docente; sin 
embargo, resaltan las limitaciones de acceso a internet y a equipos con tecnología adecuada, que propicie el uso efectivo de las TIC en el ámbito escolar. En consecuencia, el índice de favorabilidad frente a las TIC es mayor que el uso de las mismas (Rodríguez, 2004; American Association for Advancement of Science, 1998; Correa, 1999; Avendaño y Martínez, 2012). Desde el contexto de las competencias sociales se resalta cómo los niños aún prefieren la

interacción con sus amigos por el medio personal más que por el internet, lo cual se constituye en un factor positivo, porque permite valorar el uso de la internet como facilitador del aprendizaje mas no como reemplazo del encuentro presencial entre el estudiante, sus amigos y el docente, que son la esencia del acto educativo (Vygotsky, 1978; Knowles, 1982; Vander, 1986; Ausubel, 1983; Piaget, 1987; Vaillant, 2004; Villarini, 2005 y Ambrose, 2010). [fin de cita]

2. Frente a las estrategias de solución de conflictos, los estudiantes resaltan su habilidad para solicitar apoyo y ayuda, así como su actitud para evitar en lo posible las agresiones físicas; sin embargo, las categorías asociadas con agresión verbal y otro tipo de agresiones se reportan de forma relevante. Los procesos de resolución de conflictos escolares son un evento presente y prevalente en las dos instituciones educativas, aun cuando los estudiantes y los docentes reportan su intención de buscar canales y alternativas de solución pacifica que permitan, a mediano y largo plazo, reducir los niveles de agresión escolar (Restrepo, 1999),

3. Frente a los autoreportes que hacen los estudiantes y docentes, a partir de la escala MESSY se evidencia una mayor tendencia hacia el reconocimiento de sus conductas prosociales $(2,63)$, en comparación con el autoreporte de su conducta agresiva $(1,75)$. Para algunos investigadores, estos resultados podrían ser efectos de la deseabilidad social, sin embargo se destaca cómo los hallazgos de los docentes también resaltan en mayor medida las conductas prosociales que las conductas agresivas. Por lo tanto, son consistentes con las conductas de los niños (los docentes presentan un promedio de reporte de conducta prosocial de 2,45 , y un promedio de reporte de conducta agresiva de 2,04). En particular se evidenció un mayor reporte de conducta prosocial en la IE Villa Campo, aun cuando no guarda una diferencia estadísticamente significativa con la IED Carrizal. En lo referente a la observación de la conducta escolar, se sigue manteniendo una leve ventaja en la prevalencia de las conductas prosociales (con un promedio de 2,48 ), en comparación con las conductas agresivas (con un promedio de 2,26). Este último hallazgo permite validar los autoreportes, aunque las distancias entre la conductas agresivas y prosociales sean menores.

4. A partir de los análisis correlacionales establecidos entre las variables centrales del estudio, se evidencia la relación inversamente proporcional que existe entre las conductas agresivas y las conductas pro-sociales, tanto en los reportes de los estudiantes como de los docentes. Igualmente se resaltan las relaciones directamente proporcionales establecidas entre los reportes de conducta prosocial dentro los docentes, estudiantes y los registros de la institución. En la misma medida, se identificaron asociaciones directamente proporcionales entre las diferentes medidas de las conductas agresivas. Se resalta la correlación positiva o directamente proporcional establecida entre la observación de la conducta prosocial y del desempeño académico. De otra parte, el estudio también permitió identificar asociaciones estadísticamente significativas entre los hábitos de uso de internet y las preferencias por actividades que involucran el uso de TIC en la institución, así como la asociación estadísticamente significativa entre el desempeño académico y el uso de internet como medio para el desarrollo de los compromisos escolares. Finalmente, destacó la asociación estadísticamente significativa identificada entre el desempeño académico y la percepción que tienen los padres de familia sobre el papel positivo de las TIC en el proceso de aprendizaje de sus hijos e hijas.

Posterior al análisis de la información recopilada y de los resultados obtenidos, se recomienda el uso de TIC como estrategia para el desarrollo de cla- 
ses. Los resultados evidencian su rol como medio facilitadoras del aprendizaje y factor asociado al desempeño académico, tal como se evidencia en el análisis correlacional del proyecto, lo cual es congruente con los aportes de Moya (2009).

Es de suma importancia que desde la administración de las instituciones públicas se consulte e indague sobre referentes actuales, y que desde la práctica pedagógica se incursione en acciones innovadoras, aparte de las estrategias convencionales. Es relevante incursionar en modelos de flexibilización de procedimientos; la estructura administrativa debe adaptarse a los nuevos modelos de enseñanza-aprendizaje que demanda la sociedad actual (Kogan, 1971; Piaget, 1987; Sabater, 1997; De Zubiria y De Zubiria, 1994).

Estos hallazgos generan un enlace congruente con diferentes estudios de autores tales como Álvarez (1995), Amariles (2008), Espitia y Montes (2009), Avendaño y Martínez (2012), Bravo (2011), Del Rey, Ortega y Feria (2009), Hanushek y Luque (2003), Pichardo, Fernández y Amezcua (2002), Ballesteros y Cortés (2001), Simon, Lin y Gordon (1998), Yusti (1999) y Morín (1999), quienes convergen en la necesidad de profundizar en estudios que permitan analizar y comprender la complejidad de los procesos de convivencia y el desarrollo de las competencias sociales, vistos más allá del contexto del aula, como una relación sinérgica donde la familia, la sociedad y la escuela están estrechamente relacionadas y comprometidas en la generación y construcción de una nueva cultura de convivencia y desarrollo educativo integral.

\section{Referencias}

Alcaldía Municipal de Malambo (2008). Malambo Progresa: Plan de Desarrollo Municipal (2008 - 2011). Malambo: Alcaldía Municipal de Malambo.

Álvarez, A. (1995). Y la escuela se hizo necesaria. Bogotá: Magisterio.

Amar, J., Abello, R., Martínez, M., Monroy, E., Cortés, O., y Crespo, F. (2011). Categorización social y cogniciones infantiles sobre la pobreza en niños: una mirada desde el esencialismo psicológico. Investigación y Desarrollo, 19(1), 116-139. Recuperado de: http:// www.scielo.org.co/scielo.php?script $=$ sci_ arttext\&pid=S0121-32612011000100009\&lng= en\&tlng=es. .

Amariles, C. (2008). Experiencia de adaptación educativa en los niños-as del colegio básico El Dorado, que han vivido en situación de desplazamiento forzado (Tesis de grado, Universidad Católica Popular de Risaralda, Pereira).

Amarís Macías, M., Paternina Marín, A. \& Vargas Cantillo, K. (2004). Relaciones familiares en familias desplazadas por la violencia ubicadas en "la cangrejera" (corregimiento de Barranquilla, Colombia). Psicología desde el Caribe, (14) 91-124. Recuperado de http://www.redalyc.org/articulo.oa?id=21301405

American Association for Advancement of Science (1998). Aprendizaje y enseñanza efectivos (cap. 13). Recuperado el 12 de julio de 2013 de http://www.project2061.org/esp/publications/sfaa/online/chap13.htm

Colombia, Congreso de la República. Artículo 22 de 1991. Constitución Política Nacional de Colombia, 1991. Ausubel, N. (1983). Psicología educativa: Un punto de vista cognoscitivo (2. ${ }^{\mathrm{a}}$ ed.). México: Trillas.

Avendaño I., y Martínez, D. (2012). Incidencia del uso de las tecnologías de la información y la comunicación (TIC) en el desarrollo de la competencia lectora en estudiantes de 3er grado de básica primaria de una escuela pública en zona rural del municipio de Malambo (Proyecto de Investigación Index, Universidad de la Costa [CUC]).

Avendaño, I., Cortés, O., y Guerrero, H. (2013). Competencias sociales, tecnologías de la información y la comunicación TIC y desempeño académico en estudiantes con experiencia de desplazamiento forzado. Proyecto INDEX CONV-08-2013: Universidad de la Costa. Barranquilla, Colombia. 
Ballesteros, B.P. \& Cortés, O. (2001). Evaluación de las prácticas culturales y de la exposición a escenas de violencia en niños y niñas de estratos 1 y 2 de Bogotá. Suma Psicológica 8(1), 55-93

Ballesteros, B.P.; Cortés, O.F. \& Forero, D. (2001). Aplicación del modelo de ecuaciones estructurales en la explicación del comportamiento social infantil y juvenil en el estrato socioeconómico bajo de la ciudad de Bogotá. Suma Psicológica 8(1), 1-51.

Banco Mundial (2008). Datos y estadísticas. World Development Indicador on line. World Bank. Sitio WEB, [Www. bancomundial. org/: consultado el 30 de julio de 2008].

Bravo C. (2011). El bajo rendimiento escolar y los problemas de conducta. México: Colón.

Bloom, B. S.; Engelhart, M. D.; Furst, E. J.; Hill, W. H.; Krathwohl, D. R. (1956). Taxonomy of educational objectives: The classification of educational goals. Handbook I: Cognitive domain. New York: David McKay Company

Briones, G. (1999). La investigación en el aula y en la escuela. Series: Formación de docentes en investigación educativa No. 2. Bogotá DC: Convenio Andrés Bello

Chaves, A. (2001). Implicaciones educativas de la teoría sociocultural de Vigotsky. Educación 25(2), 59-65.

Coleman, J. S., Campbell, E. Q., Hobson, C. J., McPartland, J., Mood, A. M., Weinfeld, F. D., \& York, R. (1966). Equality of educational opportunity. Washington, dc, 1066-5684.

Colombia, Congreso de la República. Ley General de Educación. 115 de 1994.

Colombia, Congreso de la República. Ley 387. Recuperado el 13 de julio de 2013 de http:// www.secretariasenado.gov.co/senado/basedoc/ley/1997/ley_0387_1997.html

Colombia, Congreso de la República. Ley 1098 de 2006. Código de Infancia y Adolescencia. En Diario Oficial, n. ${ }^{\circ}$ 46-44. Correa de Molina, C.
(2004). Currículo dialógico, sistémico e interdisciplinar: Subjetividad y desarrollo humano. Bogotá: Magisterio.

Correa, C. (1999, octubre-diciembre). Construcción histórica del carácter científico de la pedagogía: Algunos referentes generales. Revista Institucional CUC, 6(20) Barranquilla.

Cubero, R. (2005). Elementos básicos para un constructivismo social. Avances en psicología latinoamericana, 23(1), 43-61. Recuperado de http://dialnet.unirioja.es/servlet/ar ticulo?codigo $=2741860$

DANE (2003). Encuesta Nacional de Calidad de Vida. Bogotá, D.C.: Departamento Administrativo Nacional de Estadística. Recuperado de: https://www.dane.gov.co/files/investigaciones/condiciones_vida/calidad_vida/Presentacion_nov25boletin.pdf

DANE (2011). Encuesta Nacional de Calidad de Vida. Bogotá, D.C.: Departamento Administrativo Nacional de Estadística. Recuperado de: https://www.dane.gov.co/files/investigaciones/condiciones_vida/calidad_vida/Presentacion_ECV_2011.pdf

De Zubiria, M., y De Zubiria, J. (1994). Biografía del pensamiento. Bogotá: Magisterio.

Del Rey, R., Ortega, R., y Feria, I. (2009). Convivencia escolar: Fortaleza de la comunidad educativa y protección ante la conflictividad escolar. Revista Interuniversitaria de Formación de Profesorado, 23(3) 159-180. Recuperado de http://www.redalyc.org/articulo. oa?id=27419066009

Delors, J., Al Mufti, I., Amagi, I., Carneiro, R., Chung, F., Geremek, B., ...Nazhao, Z. (1996). Informe a la unESCO de la Comisión Internacional sobre la Educación para el Siglo XXI: La educación encierra un tesoro. Paris: Santillana/unesco. Recuperado de http://www. secretariasenado.gov.co/senado/basedoc/ ley/1997/ley_0387_1997.html

Dunn, R., Dunn, K., \& Price, G. (1985). Manual: Learning style inventory. Lawrence, KS: Price Systems. 
Elliot, S. N., Sheridan, S. M., \& Gresham, F. M. (1989). Assessing and treating social skills deficits: A case study for the scientist-practitioner. Journal of School Psychology, 27, 197222.

Escobar, S. (2012). Bajo rendimiento académico en los estudiantes del primer grado de la escuela Rubén Darío en la comunidad del polo de desarrollo Daniel Guido Sánchez del municipio de Bluefiel, durante el primer semestre del año lectivo 2012 (Monografía, Secretaría de Educación, Managua, Nicaragua).

Espitia R., y Montes, M. (2009). Influencia de la familia en el proceso educativo de los menores del barrio Costa Azul del municipio de Sincelejo, Colombia.

Fairstein, G., y Gyssels, S. (2003). Cómo se aprende. Caracas: Federación Internacional Fe y Alegría/ Fundación Santa María.

Freire, P. (1969). La educación como práctica de la libertad. Santiago: ICIRIA.

Gardner, H. (1996). Inteligencias múltiples. Barcelona: Paidós.

Goleman, D. (1996). La inteligencia emocional: Por qué es más importante que el cociente intelectual. Buenos Aires: Javier Vergara.

Gresham, F. M., \& Cavell, T. A. (1986). Assessing adolescent social skills. En R.G. Harrington (Ed.), Testing adolescents: A reference guide for comprehensive psychological assessments (pp. 93-123). Kansas City: Test Corporation of America.

Habermas, J. (1987). Teoría y praxis: Estudios de filosofía social. Madrid: Tecnos.

Hanushek, E. A., \& J. A. Luque. (2003). Efficiency and equity in schools around the world. Economics of Education Review, 22(5), 481-502.

Helsel, W. J. \& Matson, J. L. (1984). The assessment of depression of children: The internal structure of the children's depression inventory (CDI). Behavior Research and Therapy, 22, 289-298.
Knowles, S. (1982). El estudio autodirigido: Guía para estudiantes y profesores. México: Alhambra Mexicana.

Kogan, N. (1971). Educational implications of cognitive styles. En S. Lesser (Ed.), La Psicología en la práctica educativa. México: Trillas.

Lawrence K. (1992). Psicología del desarrollo moral. Bilbao: Desclee de Brouwer.

Ledingham, J. E., \& Younger, A. J. (1985). The influence of the evaluator on assessment of children's social skills. En B. H. Schneider, K. H. Rubin, y J. E. Ledingham (Eds.), Children's peer relations: Issues in assessment and intervention (pp. 111-124). Nueva York: Springer-Verlag.

Matson, J. L., Macklin, G. F., \& Helsel, W. J. (1985). Psychometric properties of the Matson evaluation of social skills with youngsters (MESSY) with emotional problems and self-concept in deaf children. Journal of Behavior Therapy and Experimental Psychiatry, 16, 117-123.

Matson, J. L., \& Ollendick, T. H. (1988). Enhancing children's social skills. Nueva York: Pergamon Press.

Matson, J. L., Rotatori, A. F., \& Helsel, W. J. (1983). Development of a rating scale to measure social skills in children: The Matson evaluation of social skills with youngsters (MESSY). Behavior Research Therapy, 21(49), 335-340.

Mejía, M. (2006). Educación(es) en la(s) globalización(es) l: Entre el pensamiento único y la nueva crítica. Bogotá: Desde Abajo.

Merrell, K. W., \& Gimpel, G. A. (1998). Social skills of children and adolescents: Conceptualization, assessment, treatment. Mahwah, $\mathrm{NJ}$ : Lawrence Erlbaum Associates.

Ministerio de Educación Nacional. (2005). Escuela y desplazamiento. Una propuesta pedagógica (2. ${ }^{\text {a }}$ ed.). Bogotá: IMPRESOL.

Morín, E. (1999). Los siete saberes necesarios a la educación del futuro. Paris: UNESCO. [Traducción de Mercedes Vallejo-Gómez] 
Moya, A. (2009). Las nuevas tecnologías en la educación. Recuperado de http://www.csi-csif. es/andalucia/modules/mod_ense/revista/ pdf/Numero_24/ANTONIA_M_MOYA_1.pdf Consultado el 09 de diciembre de 2013

Ortega, R., y Del Rey, R. (2003). La violencia escolar: Estrategias de prevención. Barcelona: Graó

Organización de las Naciones Unidas. (1999). Declaración y programa de acción sobre una cultura de paz (Vol. 1). París: Organización de las Naciones Unidas.

Piaget, L. (1987) Etapas del desarrollo cognitivo. Ciudad: Barcelona: Seix Barral. Pichardo, M., Fernández, E., y Amezcua, J. (2002). Importancia del clima social familiar en la adaptación personal y social de los adolescentes. Revista de Psicología General y Aplicada 55 (4) [publicación en línea]. Recuperado de http://dialnet.unirioja.es/servlet/ articulo?codigo $=294345$

Restrepo, M. (1999). Escuela y desplazamiento: Una propuesta pedagógica. Bogotá: Ministerio de Educación Nacional.

Rodríguez, C. (2007). El desplazamiento forzado: Un reto para transformar la escuela (Trabajo de grado, Universidad Tecnológica de Pereira, Pereira).

Rodríguez, L. (2004). La teoría del aprendizaje significativo. Santa Cruz de Tenerife: Centro de educación a distancia.

Sabater, F. (1997). El valor de educar. Barcelona: Ariel.

Santamaría, F. (2012, agosto). Cómo aprenden los estudiantes: Siete principios para un óptimo aprendizaje [publicación en línea]. Recuperado de http://fernandosantamaria.com/ blog/2012/08/como-aprenden-los-estudiantessiete-principios-para-un-optimo-aprendizaje/

Simon, R., Lin, K., \& Gordon, L. (1998). Socialization in the family of origin and male dating violen- ce. A prospective study. Journal of Marriage and the Family, 60(1). Recuperado de: http:// www.jstor.org/stable/353862?seq=1\#page_ scan_tab_contents

Trianes, M. V., Blanca, M. J., Muñoz, A., García, B., Cardelle-Elawar, M., e Infante, L. (2002). Relaciones entre evaluadores de la competencia social en preadolescentes: Profesores, iguales y autoinformes. Anales de Psicología, 18(2) 197-214. Recuperado de http://www.redalyc.org/articulo.oa?id=16718201

Trianes, M. V., y Muñoz, A. (1989). Análisis de la percepción social en edades preescolares a través del sociométrico. En A. Jiménez y $M$. A. Lou (Eds.), II Congreso Educación y Sociedad (pp. 119-128). Granada.

Trianes, M. V., Cardelle-Elawar, M., \& Blanca, M. J. (2001). Gender, social backgrounds and self-evaluated social competence in 11-12 year old Spanish students. Documento de Trabajo. Madrid, España.

Trianes, M. V., Muñoz, A., y Jiménez, M. (1997). Competencia social: Su educación y tratamiento. Madrid: Pirámide.

Trianes, M., De la Morena, L., y Sánchez, A. (1996). Fiabilidad, componentes principales y convergencia entre diferentes medidas sociométricas y diferentes agentes evaluadores. Boletín de Psicología, 51, 1-31.

UNESCO - OREALC (2003). La educación de jóvenes y adultos en América Latina y el Caribe. Hacia un estado del Arte, Santiago de Chile: OREALC.Vaillant, D. (2004). Construcción de la profesión docente en América Latina: Tendencias, temas y debates. Santiago: oreALC.

Vander, Z. (1986). Manual de psicología social. Barcelona: Paidós.

Vargas, A. (1994), Una mirada a los conflictos Colombianos. Paris: CETRAI.

Vélaz, C. (2005). Retos de la educación básica en América Latina. Madrid: Fundación Carolina 
Villarini, A. (2005). El desarrollo del pensamiento y la pedagogía latinoamericana. Crecemos: Revista Hispanoamericana de Desarrollo Humano y Pensamiento, 3(1) [publicación en línea]. Recuperado de http://generales.uprrp. edu/pddpupr/red/revista.htm
Vygotsky, L. S. (1978). Mind in society. The development of higher psychological processes. Cambridge, MA: Harvard University Press.

Yusti, R. M. (1999). Escuela y desplazamiento. Propuesta pedagógica (2. ${ }^{\text {a }}$ ed.). Bogotá: Ministerio de Educación Nacional. 